\title{
Qualification Tests of Micro-camera Modules for Space Applications
}

\author{
By Shinichi KIMURA and Akira MIYASAKA \\ Department of Electrical Engineering, Tokyo University of Science, Noda, Japan
}

(Received November 1st, 2009)

\begin{abstract}
Visual capability is very important for space-based activities, for which small, low-cost space cameras are desired. Although cameras for terrestrial applications are continually being improved, little progress has been made on cameras used in space, which must be extremely robust to withstand harsh environments. This study focuses on commercial off-the-shelf (COTS) CMOS digital cameras because they are very small and are based on an established mass-market technology. Radiation and ultrahigh-vacuum tests were conducted on a small COTS camera that weighs less than $100 \mathrm{mg}$ (including optics). This paper presents the results of the qualification tests for COTS cameras and for a small, low-cost COTS-based space camera.
\end{abstract}

Key Words: Commercial Off-the-shelf, Micro-camera Module, Ultrahigh-vacuum Condition, Radiation

\section{Introduction}

Robust visual capability is essential for space-based activities, and recent advances in space systems have led to a concomitant increase in the complexity of operations and spacecraft systems. As a result, telemetric data and visual data are important for monitoring these systems, particularly for deployable structures and robotic operations. $^{1)-3)}$

The visual capability of a space system can also be used for sensing (e.g., star sensors and earth observation sensors) and can increase the potentialities of missions. ${ }^{4)-5)}$ Furthermore, visual information is useful for education and entertainment, particularly now that small satellites, including university satellites, are gaining popularity. In addition to current needs, unforeseen needs are likely to emerge upon the development of very small, low-cost space cameras.

Visual information is necessary for various applications. For earth observation or exploration, high reliability and high resolution are of the utmost importance. However, for situations monitoring applications, reducing cost and size is more important than improving performance in order to decrease any impact on resources. For terrestrial applications, very small cameras are continually being improved, most often for installation in mobile phones and notebook computers. However, if common terrestrial devices could be utilized in a space environment without a reduction in performance, then the cost of developing space equipment would be significantly lower than the cost of current space equipment designed specifically for use in space.

This study focuses on commercial off-the-shelf

(c) 2011 The Japan Society for Aeronautical and Space Sciences
(COTS) technologies for use in space. ${ }^{6)-9)}$ Our experience in this type of study, particularly the development of camera and image processing systems, and our knowledge of space missions have enabled us to develop low-cost space equipment using various types of COTS devices. ${ }^{10)-12)}$ In this study, we focus on COTS CMOS cameras, which are components of mobile phones and weigh less than $100 \mathrm{mg}$, including the lens.

Equipment for space applications need to clear various kinds of tests, such as thermal, vibration, radiation, and ultrahigh-vacuum. If we utilize a COTS camera module for space applications, the camera module should be qualified in terms of its structural, radiation-resistance, and thermal vacuum requirements. Since the weight of COTS camera modules is very small, their natural frequencies are very high and it is easy to cover the vibration condition. On the other hand, the radiation conditions in a space environment are rather severe for logic devices and are critically hazardous for certain types of COTS devices. The effect of space radiation can be divided into two categories: single event, and total ionizing dose. The single event is a probabilistic

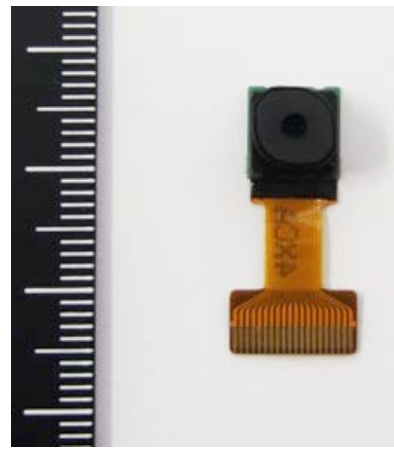

Fig. 1. MC5V.

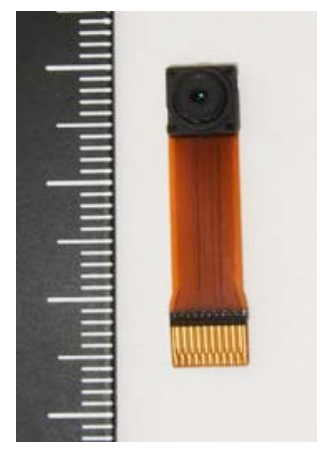

Fig. 2. NCM03-J. 
Trans. JSASS Aerospace Tech. Japan Vol. 9 (2011)

\begin{tabular}{|c|c|c|c|c|c|}
\hline & MC5V & NCM03-J & & MC5V & NCM03-J \\
\hline Size & $7.8 \times 8.0 \times 5.2 \mathrm{~mm}$ & $6 \times 6 \times 3.8 \mathrm{~mm}$ & Lenz compositio & $2 \mathrm{pcs}$ & $2 \mathrm{pcs}$ \\
\hline Weight & $0.3 \mathrm{~g}$ & & Maimum clock & $24 \mathrm{MHz}$ & $27 \mathrm{MHz}$ \\
\hline \multirow[t]{2}{*}{ Supply voltage } & \multirow[t]{2}{*}{2.5 to $2.7 \mathrm{~V}$} & \multirow{2}{*}{$\begin{array}{l}\text { Core: } 1.8 \mathrm{~V} \\
\text { IO: } 1.8 \sim 3.3 \mathrm{~V}\end{array}$} & Array size & $650 \times 503(640 \times 480)$ & 648x488(640x480) \\
\hline & & & Image area & $2.30 \times 1.72 \mathrm{~mm}$ & $2.30 \times 1.72 \mathrm{~mm}$ \\
\hline Power consumptic & $60 \mathrm{~mW}$ & $57.5 \mathrm{~mW}$ & Pixel size & $3.6 \times 3.6 \mathrm{um}$ & $4.6 \times 4.6 \mathrm{um}$ \\
\hline Field of view & $51.5 \mathrm{deg}$ & $52 \mathrm{deg}$ & Frame rate & $30 \mathrm{fps}$ & $15 \mathrm{fps}$ \\
\hline F number & 2.8 & 2.8 & Dynamic range & $48 \mathrm{~dB}$ & $62 \mathrm{~dB}$ \\
\hline Focal length (mm) & $3 \mathrm{~mm}$ & $2.32 \mathrm{~mm}$ & Sensitivity & $1.5 \mathrm{~V} /$ lux sec & $1.9 \mathrm{~V} / \mathrm{lux} \mathrm{sec}$ \\
\hline
\end{tabular}

phenomenon caused by ionized particles, which can be mitigated by software and current control. On the other hand, because total ionizing doses cause permanent degradation, weak devices cannot be utilized in situations involving them. In addition, the radiation may lead to lens degradation. Therefore, it is essential to qualify the permanent degradation effect of radiation in order to utilize a COTS camera module. Ultrahigh-vacuum conditions may also lead to permanent degradation of the lens because COTS camera modules utilize a plastic lens to reduce fabrication cost and weight. When certain types of plastics are placed in an ultrahigh-vacuum condition, the solvent contained in the plastic is released by outgassing. Based on these considerations, we decided to perform a radiation test and an ultrahigh-vacuum test on COTS camera module.

Radiation tests conducted to determine performance yielded satisfactory results throughout the tests. Furthermore, the results of ultrahigh-vacuum tests conducted on a small COTS imaging unit revealed that the imager is functional under ultrahigh-vacuum conditions, and that the lens is not damaged and does not suffer from serious outgassing. In this paper, we present the results of a qualification test conducted on COTS devices and our proposed small, low-cost COTS space camera.

\section{COTS Micro-camera Module}

We focus on two types of CMOS imagers: MC5V developed by Konica Minolta (Fig. 1) and NCM03-J developed by Asahi Electric (Fig. 2). The MC5V weighs less than $300 \mathrm{mg}$, measures $7.8 \mathrm{~mm} \times 8.0 \mathrm{~mm} \times 5.2 \mathrm{~mm}$ (including optics) and has a viewing angle of $51.5^{\circ}$. It acquires VGA resolution $(640 \times 480$ pixels $)$ images at a maximum frame rate of $30 \mathrm{fps}$. Although this resolution is not sufficiently high for detailed sensing such as for conducting earth observations, it is sufficient for in situ monitoring. The NCM03-J is much smaller than the MC5V, and weighs only $163 \mathrm{mg}$, measures $6 \mathrm{~mm} \times 6 \mathrm{~mm}$ $\times 3.8 \mathrm{~mm}$ (including optics) and has a viewing angle of $52^{\circ}$. NCM03-J also acquires VGA resolution images, but at a maximum frame rate of $15 \mathrm{fps}$. The specifications of these imagers are summarized in Table 1.

Fabrication using plastic is advantageous for the low-cost mass production of very small optics, and also allows weight reduction. Another objective of our study is to reduce the cost and size of the space camera; hence, we adopted the use of plastic optics for imaging. However,

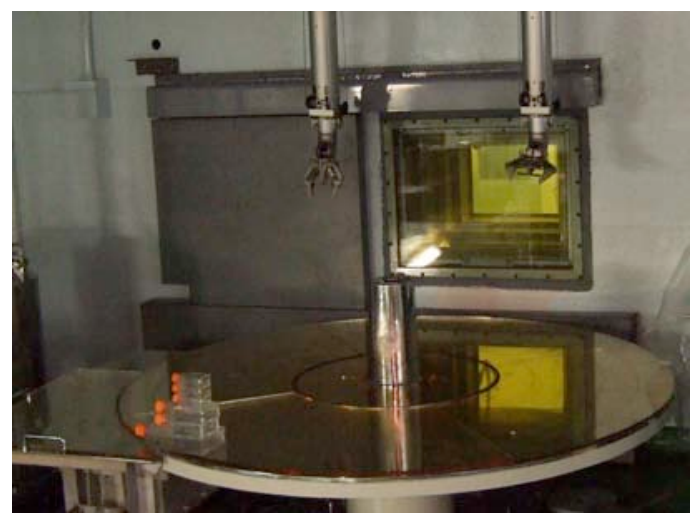

Fig. 3. Irradiation facility.

the possible degradation and contamination of plastic optics under vacuum conditions must be thoroughly investigated. ${ }^{13)}$ In addition, radiation may significantly affect both optics and electrical devices. ${ }^{14)}$ Therefore, to utilize the benefits of low-cost, lightweight devices, we investigated the performance of small COTS imagers through radiation tests and ultrahigh-vacuum tests.

\section{Radiation Tests}

To examine the performance degradation of the imagers on account of the total dose received, we irradiated them with gamma rays and compared the images acquired by the imagers both before and after irradiation. The gamma-ray irradiation test was conducted in the irradiation facility of the Department of Nuclear Engineering and Management, School of Engineering, University of Tokyo (Fig. 3), using Cs-137 as the radiation source. The source generates about $0.7 \mathrm{~Gy} / \mathrm{s}$ of radiation within a distance of $300 \mathrm{~mm}$. Figure 4 shows the apparatus used for the radiation tests. The MC5V and NCM03-J imagers were irradiated for $6 \mathrm{~h}$ from a distance of $30 \mathrm{~mm}$. The total dose, which is an integrated gamma-ray flux, corresponds to 1.5 years in a $350-\mathrm{km}$ low-Earth orbit, which is comparable to the orbit of the International Space Station. The X-rite ColorChecker ${ }^{\circledR}$ images were continuously captured during radiation.

Figure 5 shows images captured every $10 \mathrm{~min}$ before, during, and after irradiation, revealing that the image acquisition functions of both imagers were not affected by irradiation. Note that the MC5V images captured during irradiation contain white dots formed because of the high-level gamma-ray flux. The number of white dots on 

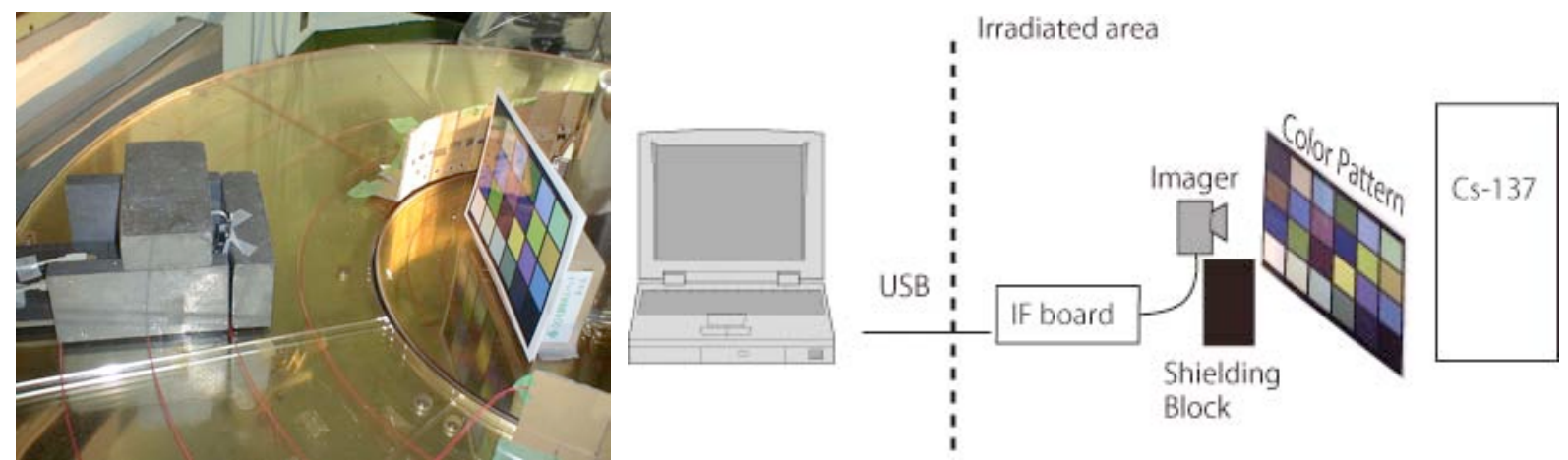

Fig. 4. Apparatus used in the irradiation experiment.

each image was about 150 on average. These types of white dots were observed when the imagers were irradiated with high radiation flux, and the number of white dots increased almost linearly with the gamma-ray flux. ${ }^{12)}$ The white dots were observed only when the imagers were irradiated. In this experiment, we increased the gamma-ray flux to 2,000 times higher than the orbital conditions in order to reduce the experimental time. We considered the white dots to be an artifact caused by the high radiation level.

The flux was increased to accelerate the irradiation in the test. For NCM03-J, it was found that the image contrast and chroma decreased with the radiation level (Fig. 5). This result demonstrates that the performance of the NCM03-J is degraded slightly because of radiation.

Degradation due to the total dose is commonly analyzed by examining the variations in electric current produced by the power supply. We found no significant variation in the electric current produced by the power supply. Figure 5 shows the raw images captured by both the imagers after irradiation. Although we found no unresponsive pixels, a decreased level of chroma and brightness was evident.
Figure 6 shows the pixel histogram of images acquired before and after the radiation test. The histogram of the MC5V is spread over the full range, and the sharpness of the peak is almost the same in both cases, even though a few peak positions are slightly shifted by the radiation. These results indicate that radiation slightly affects the contrast of the images of the MC5V. For NCM3-J, it can be seen that the resolution in chroma is somewhat lower than the MC5V because the peaks of the histogram are narrower, even before the radiation test. The higher pixels decrease in the pixel histogram after the radiation test, as represented by the red arrows. This type of decrease is typical in blue pixels; therefore, the images darken and the color balance shifts to red.

The results suggest that both the imagers are functional under irradiation conditions, and are therefore suitable for orbital applications, although their performance decreases slightly under these conditions. We tested the MC5V further under vacuum conditions because it performed much better than the NCM03-J.

\section{MC5V}
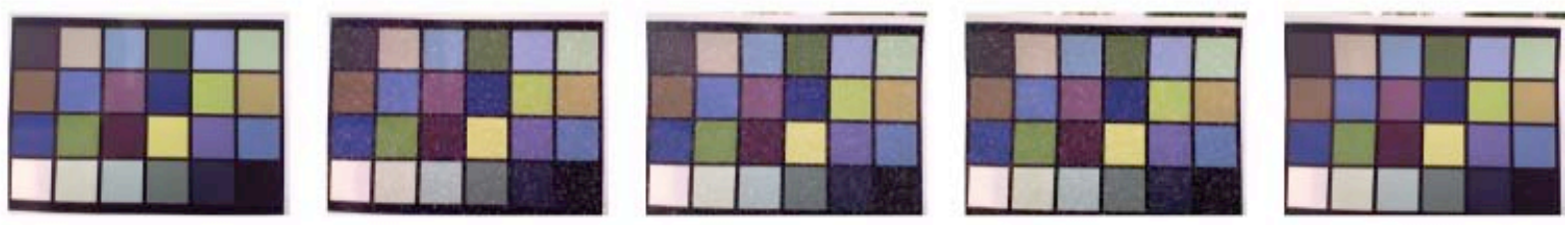

\section{NCM3-J}

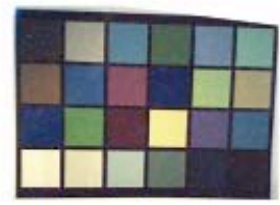

Before

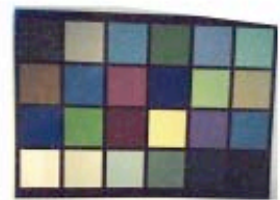

$10 \mathrm{~min}$

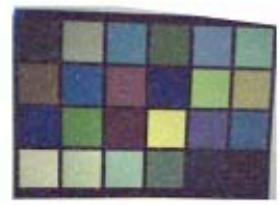

3 hours

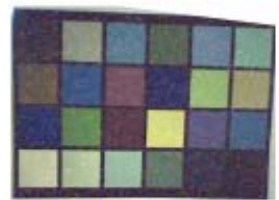

6 hours

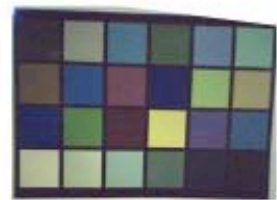

After

Fig. 5. Images acquired in the radiation test. 
(a)MC5V

RGB

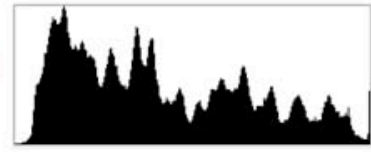

R

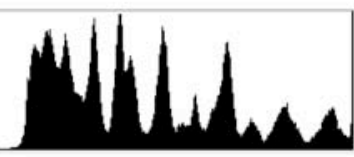

G

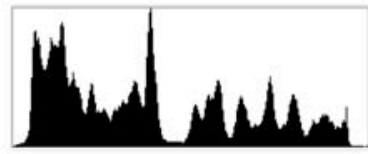

B

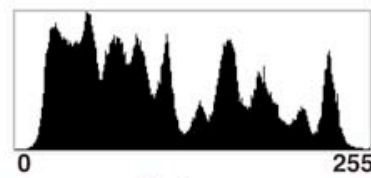

Before
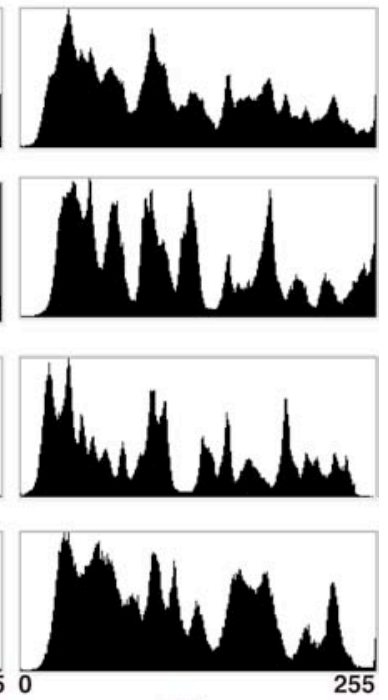

After
(b)NCM3-J
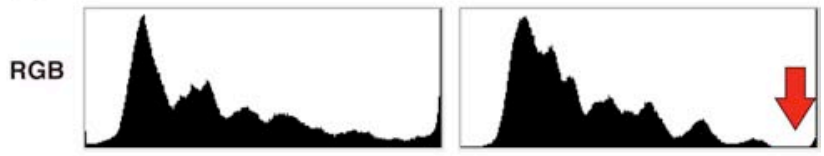

R
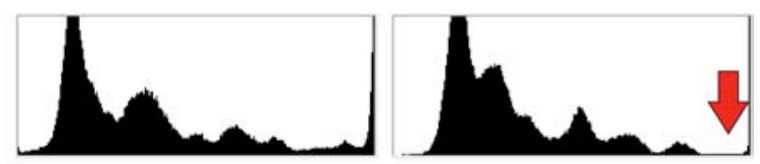

G

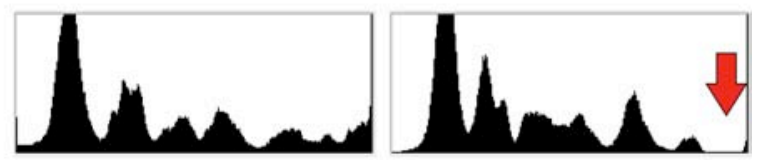

B

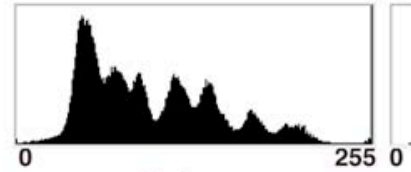

Before

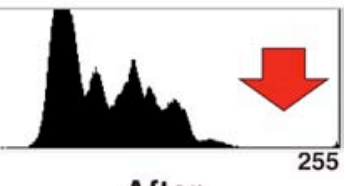

After

Fig. 6. Pixel histograms of acquired images before and after the radiation test.

\section{Ultrahigh-vacuum Tests}

To examine the degradation characteristics of plastic optics caused by ultrahigh-vacuum conditions, we conducted vacuum tests on three MC5V units using the ultrahigh-vacuum chamber at the Shimizu Institute of Technology with the cooperation of Shimizu Corporation. For these tests, we focused only on the static degradation of plastic optics. We therefore compared images acquired before and after applying ultrahigh-vacuum conditions, without applying any power or signal to the imagers.

Three MC5V units were subjected to an ultrahigh-vacuum of $10^{-7}$ Torr for $40 \mathrm{~h}$.

On the color pattern images acquired before and after the application of the ultrahigh-vacuum condition, we found that the color levels in these images were essentially the same, and that the chroma or brightness levels did not change.

We used a calibration panel to analyze and compare the image center and focal length both before and after applying the ultrahigh-vacuum condition. Figure 7(a) shows the change in focal length. The focal length may have increased slightly after applying the ultrahigh-vacuum condition. However, if we consider that the analyzing method using a calibration panel had a measurement error of \pm 0.1 pixel, and that the changes in the control lens, which was not placed under the ultrahigh-vacuum condition, were of the same order, changes as the result of being subjected to an ultrahigh-vacuum were not significant. Figure 7(b) shows the change in the image center, where $(0,0)$ represents the ideal image center, in this case $(320,240)$, and each point represents the shift from the ideal image center in pixels. For the situation monitoring, which is considered to be the major target application of the camera module, only recognition of abstract shapes is required. Therefore, if focus is not significantly affected and the focused image is obtained, there is no explicit requirement for geometric determine only the degradation of optics. The blue

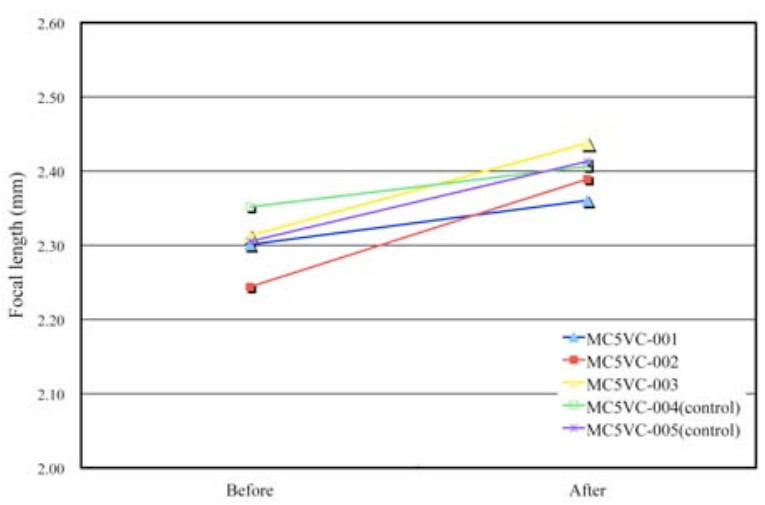

(a) Changes in focal length.

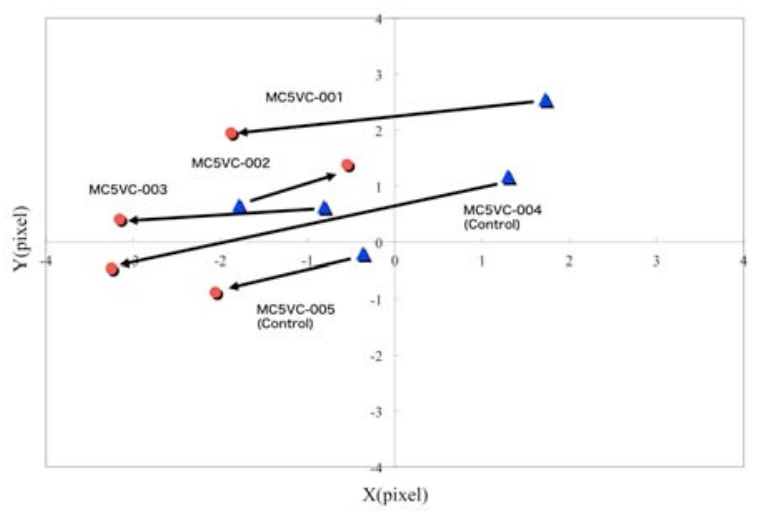

(b) Changes in focal center.

Fig. 7. Changes in camera parameters. 
triangles represent the shift in position of the image center prior to applying the ultrahigh-vacuum condition, while red circles represent the shift after application. The similarities between the images before and after application of the condition are represented by arrows, with the sample names noted next to them. That is, for MC5VC-001, the image center on the $x$-axis changed from +2 , corresponding to the 322 pixel position, to -2 on the $y$-axis, corresponding to the 318 pixel position. These parameters were measured by image-processing software using a calibration board image captured by the imager because the lens and imager cannot distinguish between modular cameras. Therefore, the measurement error was rather large (around 1 pixel). There were no specific trends observed before or after applying the ultrahigh-vacuum condition. When considering that situation monitoring is the major application of these module cameras, we did not feel that these shifts will have an effect on practical applications. Based on the aforementioned considerations, the results suggest that the effect of an ultrahigh-vacuum condition on camera parameters is not significant.

To determine the amount of outgassing, we compared the weights of the MC5V units before and after subjecting them to the ultrahigh-vacuum condition. The results are summarized in Fig. 8. Changes in weight after applying the ultrahigh-vacuum condition were not significant because the measurement error of the scale is $0.03 \mathrm{~g}$. Outgassing may be hazardous in two ways: one is contamination of other equipment, and the other is degradation of the camera itself. Because the total mass of the camera modules is negligible, contamination of other equipment will also be very small, even if outgassing comparable to the measurement error of $0.03 \mathrm{~g}$ occurs. Additionally, a significant degradation of optics was not observed in these optical experiments. These results suggest that the effect of MC5VC outgassing is negligible.

From these results, we conclude that the MC5V imager is suitable for orbital applications.

\section{Conclusion}

The visual capability of a space system is crucial for space activities. We studied COTS CMOS imagers, which are mass-produced mobile phone components, and conducted ultrahigh-vacuum tests and radiation tests on an MC5V imager, which is a small COTS imaging unit. The weight of this unit, including optics, is less than $100 \mathrm{mg}$.

The results of testing indicated that an MC5V imager is suitable for space applications. In addition, we found that outgassing from the imager was small and that contamination was negligible, which we attribute to the low volume of plastic used in the imager. We are currently developing a very small, low-cost space camera on the basis of these results.

\section{Acknowledgments}

The authors wish to thank Dr. Yokota, of the

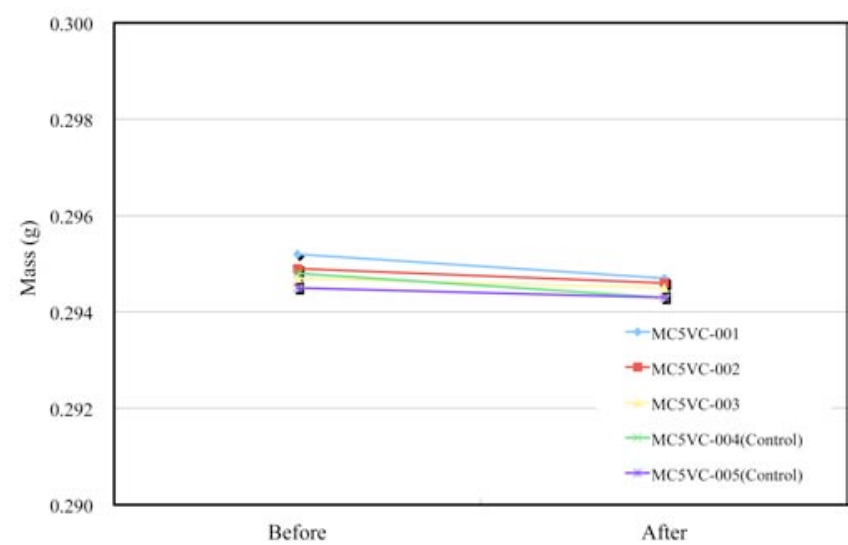

Fig. 8. Mass change in vacuum condition test.

University of Tokyo School of Engineering, and Dr. Eguchi, of the University of Tokyo Department of Nuclear Engineering and Management, School of Engineering, for their help with the radiation tests.

We are also grateful to Dr. Fukase, Dr. Sato and Dr. Yoshida of Shimizu Corporation for their support in the vacuum tests.

\section{References}

1) Kimura, S., et. al.: Visual Analysis in a Deployable Antenna Experiment using Sub-pixel Cross-correlation, IEEE Transactions on Aerospace and Electronic Systems, 40 (2004), pp. 247-258.

2) Suzuki, Y., et. al.: Mechanism for Assembling Antenna in Space Development and Verification on Engineering Test Satellite VII -, IEEE Transactions on Aerospace and Electronic Systems, 37 (2001), pp. 254-265.

3) Kimura, S., et. al.: Robot-aided Remote Inspection Experiment on STS-85, IEEE Transactions on Aerospace and Electronic Systems, 36 (2000), pp. 1290-1297.

4) Eisenman, A., Joergensen, J. L., Liebe, C. C.: Real Sky Performance of the Prototype Ørsted Advanced Stellar Compass, Proceedings of Aerospace Applications Conference, Aspen, U.S.A., Vol. 2, 1996, pp. 103-113.

5) Kimura, S., et. al.: Rendezvous Experiments on SmartSat-1, Space Mission Challenges for Information Technology (SMC-IT 2006), Pasadena, CA, USA, 2006, pp. 374-379.

6) Sasada, T.: In-Flight Measurement of Space Radiation Effects on Commercial DRAM, The 16th International Conference on Microelectronics, 2004, 1434175.

7) Goka, T., et. al.: Space Environment \& Effect Measurements From the MDS-1 (Tsubasa) Satellite, Proceedings of International Symposium on Space Technology and Science, Matsue, Japan, ISTS 2002-h-32p, 2002.

8) Winokur, P. S., et. al.: Use of COTS Microelectronics in Radiation Environments, IEEE Transactions on Nuclear Science, 46 (1999), pp. 1494-1503.

9) Underwood, C. I. and Oldfield, M. K.: Observed Radiation-Induced Degradation of Commercial-Off-The-Shelf (COTS) Devices Operating in Low-Earth Orbit, IEEE Transactions on Nuclear Science, 45 (1998), pp. 2737-2744.

10) Kimura, S., et al.: Preliminary Experiments on Technologies for Satellite Orbital Maintenance Using Micro-LabSat 1, Advanced Robotics, 18 (2004), pp. 117-138.

11) Kimura, S., et al.: Single-Event Performance of a COTS-Based MPU under Flare and Non-Flare Conditions, IEEE Transactions on Aerospace and Electronic Systems, 41 (2005), pp. 599-607.

12) Kimura, S., et al.: Experiments on High Performance Image Processing Technologies on Micro-LabSat, 54th International 
Trans. JSASS Aerospace Tech. Japan Vol. 9 (2011)

Astronautical Congress, 2003, Bremen, Germany, IAC-03-IAA.11.2.04.

13) Saito, M., Anbai, K., Hayashi, T.: Organic Outgassing Behavior of Plastic Material and Reduction of Organic

Contamination in Semiconductor Equipment, IEEE Transactions on Semiconductor Manufacturing, 18 (2005), pp. $575-583$

14) Lee, P. P. K., Thompson, D. A., Modney, D. L.: Total
Ionizing Dose Gamma and Proton Radiation Testing on a COTS Interline $\mathrm{CCD}$ with Microlens, Proceedings of Radiation Effects Data Workshop, 2005, pp. 163-169. 